\title{
Unified Equivalent-circuit Models for Voltage-source Inverters that Capture Averaged Dynamics and Power-flow Solutions in Distribution Networks
}

\author{
Minghui Lu \\ University of Washington \\ mhlu@uw.edu
}

\author{
Victor Purba \\ University of Minnesota \\ purba002@umn.edu
}

\author{
Sairaj Dhople \\ University of Minnesota \\ sdhople@umn.edu
}

\author{
Brian Johnson \\ University of Washington \\ brianbj@uw.edu
}

\begin{abstract}
This paper demonstrates how three-phase distribution networks composed of voltage-source inverters can be modeled as a single unified equivalent-circuit network realized with familiar circuit elements. Such a model is derived by representing all physical-and control-subsystem dynamics as equivalent circuits. Two versions are put forth: the first captures averaged dynamics; while the second is a steady-state version of the first and it captures the power-flow solution in sinusoidal steady state. The main challenge in undertaking such an effort is presented by the fact that inverters are composed of subsystems (filters, pulse width modulators, phase-locked loops, controllers, direct-quadrature reference-frame transformations) that belong to multiple domains (physical and control). We demonstrate how all these constituent subsystems can be transcribed as equivalent circuits which then promote a single and unified circuit model that captures network physical-and control-layer dynamics. Numerical simulations for a representative distribution network compare results from the averaged model and the steady-state model with high-fidelity switch-level simulations. The results establish the validity of the circuit-based models and the computational benefits of the proposed approach.
\end{abstract}

\section{Introduction}

Power systems are rapidly transforming due to the integration of inverter-interfaced resources. Multi-inverter ac power systems are characterized by complex dynamics that present a variety of challenges with respect to modeling and analysis. Conventionally, such systems are analyzed with models that clearly distinguish dynamics attributable to different domains.

This material is based upon work supported in part by the U.S Department of Energy's Office of Energy Efficiency and Renewable Energy (EERE) under Solar Energy Technologies Office (SETO) Agreement Number EE0009025. Brian Johnson was also supported by the Washington Research Foundation.
In other words, there is a clear distinction made between the physical layer and the controllers [1-3]. While this is representative of implementation, it promotes a disjoint viewpoint that impedes a holistic and system-level appreciation of operation across timescales. This paper departs from the convention of distinguishing domain-specific models and puts forth a modeling paradigm that is wholly centered on equivalent circuits. In particular, we demonstrate how networks composed of inverters can be cohesively represented as a single and unified collection of interconnected circuit elements. The fundamental challenge involved in obtaining such a unified system model is to translate the complex control-layer dynamics pertinent to inverters (spanning coordinate transformations, current-control loops, pulse-width modulation, phase-locked loops) as equivalent circuits. By addressing this challenge, the resulting circuit-based model is seamlessly unified and it dissolves the boundary between physical- and control-layer dynamics. It can be assembled quite simply with circuit elements (inductors, capacitors, resistors, dependent and independent current and voltage sources) that are native to circuit simulators. Such a model promotes a unified analytical examination of complex networks involving inverters leveraging circuit theory. In addition, it naturally yields a surrogate simplified model for steady-state analysis that can recover the power-flow solution.

There is a rich history of equivalent circuits being leveraged in sub-disciplines of power engineering. The most prominent example that springs to mind is the wide body of work on magnetic equivalent circuits wherein circuit models are formulated to capture the evolution of fluxes and fields in magnetic components [4-6]. Equivalent-circuit models that bridge the gap between mechanical and electrical domains for electromagnetic interfaces such as synchronous generators and induction machines are quite common [7-10]. (Notably, what is not common in such systems are models that also describe companion controllers as circuits.) Shifting focus to power electronics, equivalent-circuit models 
for open-loop converters are well established [11-13]. More recently, circuit models that attempt to capture closed-loop operation of power electronics circuits have been established with so-called impedance-based analysis [14-18]. The output terminals of a converter in such a framework are interpreted as a Thévenin or Norton lumped two-element equivalent circuit. Unfortunately, such lumped models absorb and obscure the internal structure of the compensator, nonlinearities, as well as feedback and feedforward paths. On another related note, nonlinear circuits have recently been featured prominently in the control of grid-forming inverters [19-24]; in such systems, system-level circuit models for the distribution network (of the type we outline in this work) would follow similarly.

Closest in spirit to the direction we pursue in this work are the efforts in $[25,26]$ which demonstrated how models for closed-loop voltage- and current-controlled power-electronics circuits can be represented with elementary $R L C$ components, and dependent voltage and current sources. In particular, the work in [25] focuses on dc-dc converters whereas the results in [26] establish an equivalent-circuit model for a single current-controlled inverter in a synchronously rotating reference frame. Building on $[25,26]$, this work provides the following unique contributions:

- A network-level equivalent-circuit model is put forth to describe physical- and control-layer dynamics of three-phase distribution networks comprising current-controlled voltage-source inverters. This circuit yields averaged dynamics of network- and inverter-level voltages, currents, and control signals.

- A steady-state version of the above model (that does not involve inductive and capacitive elements) follows as a direct extension and facilitates the computation of power-flow solutions in such distribution networks. This version can be utilized to initialize complex switched-level simulations with ease.

The two system-level circuit models outlined above offer a modeling and simulation strategy that departs from the norm of multi-domain simulations that are common practice in inverter-dominant power networks. In particular, typical approaches to model and simulate inverter networks are individually tailored and customized for handling dynamic excursions and steady-state separately. Our formulation presents a unified strategy that significantly simplifies analysis across timescales. Furthermore, for steady-state analysis, the equivalent-circuit approach yields the solutions of network-level and internal inverter states in one step. This is a pronounced departure from applying common power-flow formulations which would first yield inverter terminal voltages as a solution, from which the internal states are worked out separately. We also note that the same simulation software package that returns the solution of the averaged dynamics can be readily modified to yield the solution of the steady-state model in one time step of the simulation. As such, one can switch back and forth between the two with ease or simulate different parts of the network with different temporal fidelity.

The main challenge that we address in deriving the models referenced above is to depict reference-frame transformations (between three-phase and direct-quadrature coordinates) and phase-locked loops as equivalent circuits. In particular, we show that the two-dimensional rotation transformation between any two arbitrary orthogonal (i.e., direct-quadrature) frames can be captured via interconnected ideal transformers with turns ratios that are a function of the angular difference between the reference frames. Power conservation in coordinate transformations is naturally aligned with the behavior of ideal lossless transformers. A key next step is to couple the dynamics of the phase-locked loop with the ideal transformers discussed above to close the loop. The resulting circuit for the voltage-source inverter captures the operation of all physical and control systems in the local direct-quadrature reference frame; furthermore, it is readily interfaced to the external distribution network that is modeled in a global direct-quadrature reference frame.

The remainder of this paper is organized as follows. The original switched-level dynamic models for the inverter physical and control subsystems are overviewed in Section 2. Accompanying the discussion for each subsystem, we provide the equivalent-circuit models that capture averaged dynamics and steady-state operation. Numerical simulations for an illustrative distribution network compare results from a high-fidelity switched simulation with those from the equivalent-circuit averaged model in Section 3. We also verify that the steady-state operating point can be recovered from the snapshot solution of the corresponding steady-state equivalent-circuit model. Concluding remarks and a few directions for future work are provided in Section 4.

\section{Three-phase Inverter Model and Equivalent-circuit Representation}

In this section, we outline the dynamical model for the grid-tied three-phase inverter. We consider 


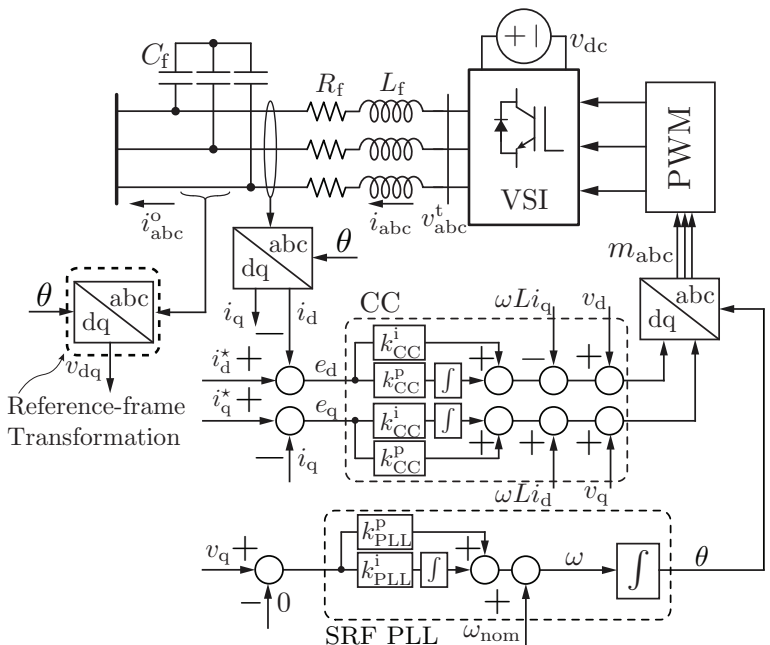

(a) switched model

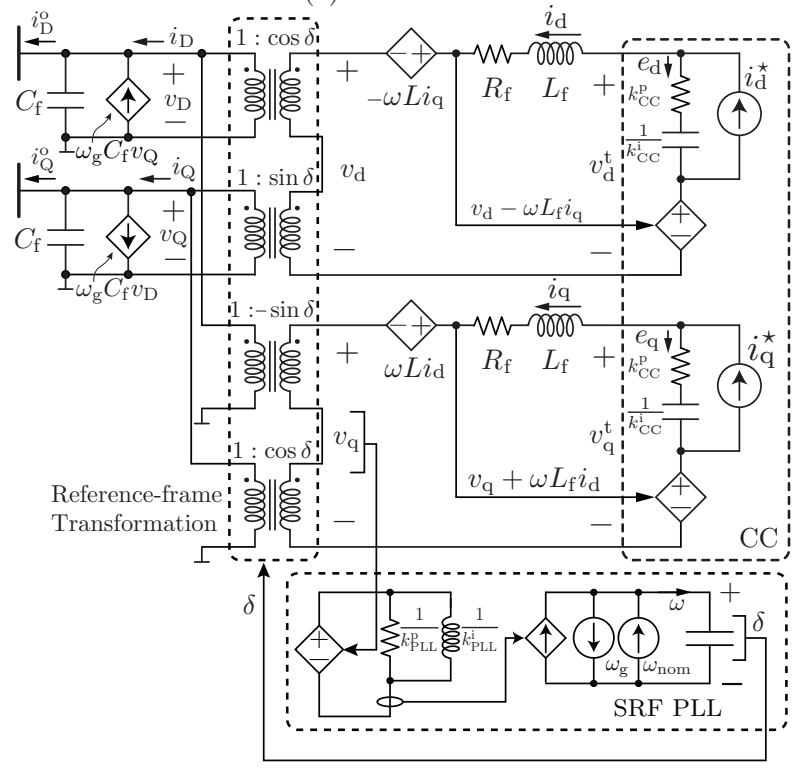

(b) averaged model

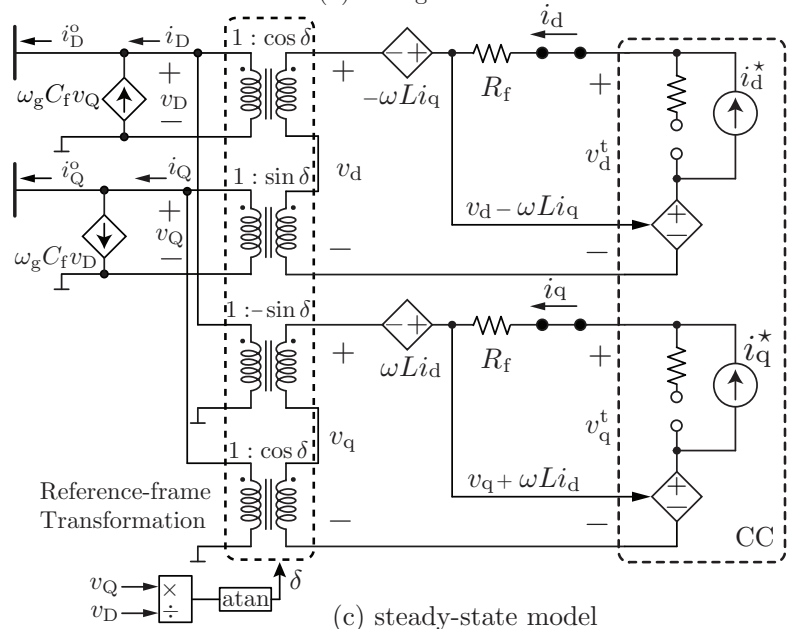

(c) steady-state model

Figure 1: (a) Switched model and equivalent-circuit models capturing (b) averaged and (c) steady-state operation.

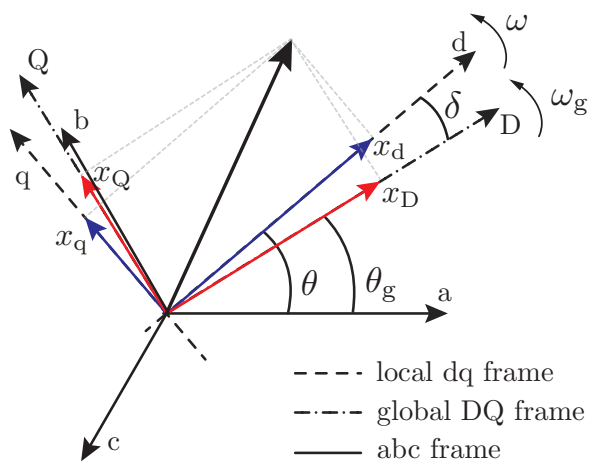

Figure 2: Three-phase abc waveforms are be represented in the global (network) DQ and local (inverter) dq reference frames. Signals in the global DQ-frame $\left(x_{\mathrm{D}}, x_{\mathrm{Q}}\right)$ are indicated by red vectors, while those in the local dq-frame $\left(x_{\mathrm{d}}, x_{\mathrm{q}}\right)$ are in blue.

a voltage source inverter (VSI) with an output $L C$ filter. The control architecture contains a current controller, a pulse-width modulation (PWM) block, a three-phase to dq transformation, and a phase-locked loop (PLL). See Fig. 1(a) for an illustration. This is a prototypical implementation of the control architecture for voltage-source inverters and has been frequently referenced in similar forms [27-30].

Accompanying the description of the inverter control- and physical-layer dynamics, we present equivalent circuits that capture the averaged dynamics and the steady-state behavior of the inverter in a suitable direct-quadrature reference frame. (See Figs. 1(b) and 1(c), respectively.) We emphasize that signals in the averaged model (Fig. 1(b)) are anticipated to evolve with time but not sinusoidally since the driving terms are dc sources. On the other hand, signals in the steady-state circuit (Fig. 1(c)) are expected to be purely dc. In each case, corresponding sinusoidal waveforms can be recovered by applying a suitable inverse transformation. (See Section 2.1 for details.)

The inverters that we examine will be assumed to be interconnected via transmission lines modeled as series $R L$ circuits in a balanced three-phase distribution network. One node of this distribution network, g, represents the point of its interconnection with the remainder of the bulk power grid and is modeled as an infinite bus with fixed voltage magnitude, frequency, $\omega_{\mathrm{g}}$, and phase angle, $\theta_{\mathrm{g}}$. We provide further details about the network-level models in Section 3.

\subsection{Reference-frame Transformation}

To facilitate analysis, we transform all three-phase signals to stationary (dc) signals in suitable 
direct-quadrature (dq) reference frames. We focus on two stationary reference frames. Network-related quantities are represented in a so-called global DQ reference frame using Park's transformation [27]. Given three-phase network-related signals $x_{\mathrm{a}}, x_{\mathrm{b}}, x_{\mathrm{c}}$, this involves generating signals $x_{\mathrm{D}}, x_{\mathrm{Q}}$ via:

$$
\left[\begin{array}{l}
x_{\mathrm{D}} \\
x_{\mathrm{Q}}
\end{array}\right]=\frac{2}{3}\left[\begin{array}{cc}
\cos \theta_{\mathrm{g}} & -\sin \theta_{\mathrm{g}} \\
\cos \left(\theta_{\mathrm{g}}-\frac{2 \pi}{3}\right) & -\sin \left(\theta_{\mathrm{g}}-\frac{2 \pi}{3}\right) \\
\cos \left(\theta_{\mathrm{g}}+\frac{2 \pi}{3}\right) & -\sin \left(\theta_{\mathrm{g}}+\frac{2 \pi}{3}\right)
\end{array}\right]^{\mathrm{T}}\left[\begin{array}{c}
x_{\mathrm{a}} \\
x_{\mathrm{b}} \\
x_{\mathrm{c}}
\end{array}\right]
$$

On the other hand, individual inverter controllers are modeled in local dq reference frames that are established based on the angle, $\theta$, corresponding to the PLL of each inverter. (See Fig. 1(a).) All network-related signals are sensed and transformed to local reference-frame representations as shown in the system architecture sketch in Fig. 1(a). In particular, this involves the application of Park's transformation (1) with PLL angle $\theta$. For the purpose of modeling, we will find it useful to define

$$
\delta=\theta-\theta_{\mathrm{g}}
$$

which allows us to define the coordinate transformation between the global DQ to local dq reference frames as

$$
\left[\begin{array}{l}
x_{\mathrm{d}} \\
x_{\mathrm{q}}
\end{array}\right]=\left[\begin{array}{cc}
\cos \delta & \sin \delta \\
-\sin \delta & \cos \delta
\end{array}\right]\left[\begin{array}{l}
x_{\mathrm{D}} \\
x_{\mathrm{Q}}
\end{array}\right] .
$$

One motivation to develop models that emphasize relative global-to-local angle differences is that $\delta$ settles to a dc value in steady state. Figure 2 illustrates the different reference frames discussed above.

Equivalent-circuit Representation. Given that the coordinate transformation in (3) is power invariant, it is possible to represent this rotation matrix as a circuit composed of ideal transformers. In particular, this involves ideal transformers with their primary side connected to the network (represented in the global DQ frame) and their secondary side interfaced to inverter variables (represented in the local dq frame). Such interconnections replicate the matrix operations in (3). See segments labelled Reference-frame Transformation in Figs. 1(b)-1(c). From (3), one can work out that the transformers must have the primary terminals connected in parallel, and the secondary terminals connected in series. For the d-axis circuit, the turns ratios of the transformers are $1 / \cos \delta$ and $1 / \sin \delta$, respectively; while for the q-axis circuit, the turns ratios are $-1 / \sin \delta$ and $1 / \cos \delta$, respectively.

\subsection{Phase-locked Loop (PLL)}

We consider a classical synchronous reference frame PLL (SRF-PLL). It operates in the local inverter dq reference frame and consists of a PI controller with PI gains $k_{\mathrm{PLL}}^{\mathrm{p}}, k_{\mathrm{PLL}}^{\mathrm{i}}$. (See Fig. 1(a) for implementation.) The SRF-PLL tracks the angle at the point of common coupling of each inverter with the network; in the setting we consider, this corresponds to the angle of the voltage at the capacitor $C_{\mathrm{f}}$ of the output $L C$ filter. The dynamics of the PLL angle $\theta$ are given by

$$
\dot{\theta}=\omega_{\text {nom }}-k_{\mathrm{PLL}}^{\mathrm{p}} v_{\mathrm{q}}-k_{\mathrm{PLL}}^{\mathrm{i}} \int v_{\mathrm{q}} d t
$$

where the feedforward term $\omega_{\text {nom }}=2 \pi \times 60[\mathrm{rad} / \mathrm{sec}]$ biases operation close to the expected nominal grid frequency. Notice that the dynamics (4) attempt to drive the q-axis voltage, $v_{\mathrm{q}}$, asymptotically to zero. It can be shown that $v_{\mathrm{q}}=0$ implies $\theta$ corresponds to the phase-a voltage of the filter capacitance, $C_{\mathrm{f}}$, while its derivative, $\dot{\theta}=: \omega$, corresponds to the electrical frequency of the network, $\omega_{\mathrm{g}} \cdot{ }^{1}$

Equivalent-circuit Representation. An equivalent circuit for the SRF-PLL should accept the filter-capacitance q-axis voltage, $v_{\mathrm{q}}$, as input, and return angle $\delta$ as output. Even though the PLL angle is $\theta$, for the purpose of integrating circuit models across subsystems with finite electrical signals, we focus on reference-frame transformations with angle $\delta$ to obtain the equivalent-circuit models. (Notice, e.g., that in the same spirit, the transformers capturing the DQ-dq reference-frame transformations are formulated with turns ratios depending on $\delta$.) Segments labelled $S R F-P L L$ in Figs. 1(b)-1(c) illustrate the equivalent-circuit models for the PLL capturing the averaged dynamics and steady-state operation, respectively. A few points deserve emphasis. For the model in Fig. 1(b) corresponding to the averaged dynamics, the PI controller is equivalent to a parallel connection between a voltage source that represents the input to the controller, a resistor $1 / k_{\mathrm{PLL}}^{\mathrm{p}}$, and inductor $1 / k_{\text {PLL }}^{\mathrm{i}}$. The current injection into the unit-value capacitance (alongside other current sources capturing $\left.\omega_{\text {nom }}, \omega_{\mathrm{g}}\right)$ represents the PLL frequency, $\omega$. The voltage that develops across the unit-value capacitance is equal to $\delta$. For the model in Fig. 1(c) corresponding to steady-state operation, we simply recognize that $\delta=$ $\arctan \left(v_{\mathrm{Q}} / v_{\mathrm{D}}\right)$ is the steady-state value and leverage

\footnotetext{
${ }^{1}$ Suppose $v_{\mathrm{a}}=V \cos \theta_{\mathrm{g}}=V \cos \omega_{\mathrm{g}} t$. Assuming balanced three-phase operation, Park's transformation in (1) with angle $\theta$ yields $v_{\mathrm{q}}=V \sin \left(\theta_{\mathrm{g}}-\theta\right)$. We see that $v_{\mathrm{q}}=0$ implies $\theta=\theta_{\mathrm{g}}$. Furthermore, we get $\omega:=\dot{\theta}=\dot{\theta}_{\mathrm{g}}=\omega_{\mathrm{g}}$.
} 
this to realize the turns ratios in the reference-frame transformers directly.

\subsection{Output Filter}

In this section, we focus on the output $L C$ filter of the inverters. For simplicity of notation and without loss of generality, we will find it useful to express the current in the inductive branch of the filter in the local dq reference frame, and the voltage across the capacitive branch in the global DQ frame. The dynamics of the filter are then given by:

$$
\begin{aligned}
& {\left[\begin{array}{l}
\dot{i}_{\mathrm{d}} \\
i_{\mathrm{q}}
\end{array}\right]=\left[\begin{array}{cc}
-\frac{R_{\mathrm{f}}}{L_{\mathrm{f}}} & \omega \\
-\omega & -\frac{R_{\mathrm{f}}}{L_{\mathrm{f}}}
\end{array}\right]\left[\begin{array}{c}
i_{\mathrm{d}} \\
i_{\mathrm{q}}
\end{array}\right]+\frac{1}{L_{\mathrm{f}}}\left[\begin{array}{c}
v_{\mathrm{d}}-v_{\mathrm{d}}^{\mathrm{t}} \\
v_{\mathrm{q}}-v_{\mathrm{q}}^{\mathrm{t}}
\end{array}\right],} \\
& {\left[\begin{array}{c}
\dot{v}_{\mathrm{D}} \\
\dot{v}_{\mathrm{Q}}
\end{array}\right]=\left[\begin{array}{cc}
0 & \omega_{\mathrm{g}} \\
-\omega_{\mathrm{g}} & 0
\end{array}\right]\left[\begin{array}{l}
v_{\mathrm{D}} \\
v_{\mathrm{Q}}
\end{array}\right]+\frac{1}{C_{\mathrm{f}}}\left[\begin{array}{c}
i_{\mathrm{D}}-i_{\mathrm{D}}^{\mathrm{o}} \\
i_{\mathrm{Q}}-i_{\mathrm{Q}}^{\mathrm{O}}
\end{array}\right],}
\end{aligned}
$$

where $\left(v_{\mathrm{d}}^{\mathrm{t}}, v_{\mathrm{q}}^{\mathrm{t}}\right)$ denotes the voltage at the inverter switched terminals in the local dq frame, and $\left(i_{\mathrm{D}}^{\circ}, i_{\mathrm{Q}}^{\circ}\right)$ denotes the current injected into the network expressed in the global DQ frame. See Fig. 1(a) for an illustration.

Equivalent-circuit Representation. The averaged and steady-state equivalent-circuit models for the output $L C$ filter follow from (5)-(6). Notice that the filter capacitor is situated on the primary-side of the transformer modeling the reference-frame transformation since its dynamics are written in the global DQ reference frame. On the other hand, the filter inductor is situated on the secondary-side since its dynamics are modeled in the local dq reference frame. In both the averaged and steady-state equivalent circuits, we have dependent-voltage and dependent-current sources that arise from the off-diagonal terms in the system matrices of (5)-(6). For the steady-state circuits, we simply short circuit inductors and open circuit capacitors.

\subsection{Current Controller (CC)}

The current controller is composed of two PI controllers with PI gains $k_{\mathrm{CC}}^{\mathrm{p}}, k_{\mathrm{CC}}^{\mathrm{i}}$ and feedforward terms to cancel the cross-coupling terms discussed earlier in the description of the output filter. The inputs to the controller are the current references, $i_{\mathrm{d}}^{\star}$ and $i_{\mathrm{q}}^{\star}$.
The outputs are the voltage reference terms, given by:

$$
\begin{gathered}
v_{\mathrm{d}}^{\star}=v_{\mathrm{d}}+\omega L_{\mathrm{f}} i_{\mathrm{q}}+k_{\mathrm{CC}}^{\mathrm{p}}\left(i_{\mathrm{d}}^{\star}-i_{\mathrm{d}}\right) \\
+k_{\mathrm{CC}}^{\mathrm{i}} \int\left(i_{\mathrm{d}}^{\star}-i_{\mathrm{d}}\right) d t . \\
v_{\mathrm{q}}^{\star}=v_{\mathrm{q}}-\omega L_{\mathrm{f}} i_{\mathrm{d}}+k_{\mathrm{CC}}^{\mathrm{p}}\left(i_{\mathrm{q}}^{\star}-i_{\mathrm{q}}\right) \\
+k_{\mathrm{CC}}^{\mathrm{i}} \int\left(i_{\mathrm{q}}^{\star}-i_{\mathrm{q}}\right) d t .
\end{gathered}
$$

Equivalent-circuit Representation. Following a similar strategy as before, equivalent-circuit models corresponding to the current controller for averaged dynamics and steady-state operation can be derived and these are labelled $C C$ in Figs. 1(b)-1(c), respectively. In the averaged dynamic model illustrated in Fig. 1(b), the PI compensator is represented by an $R C$ circuit with resistance $k_{\mathrm{CC}}^{\mathrm{p}}$ and capacitance $1 / k_{\mathrm{CC}}^{\mathrm{i}}$ fed by current sources $i_{\mathrm{d}}^{\star}$ and $i_{\mathrm{q}}^{\star}$. The dependent-voltage sources capture the feedforward terms in (7)-(8). The equivalent circuit for steady-state operation is recovered simply by open circuiting the capacitors corresponding to the compensators.

\section{Simulation Case Study for Representative Distribution Network}

In this section, we delineate the overall power system model and how the network-level model interfaces to that for individual inverters. Ideas are grounded in the context of a particular representative three-phase distribution network. Following this, we present simulation results to validate the proposed models.

\subsection{Network Description}

Consider the balanced three-phase network with three grid-tied three-phase inverters shown in Figure 3(a). The network is composed of interconnecting lines modeled as series $R L$ circuits, and an infinite bus which represents the transmission-distribution interconnection. For simplicity, the impedances of all branches in the distribution network are assumed to be identical. The three-phase abc voltages at the infinite bus are denoted by $E_{\mathrm{a}}, E_{\mathrm{b}}, E_{\mathrm{c}}$, and the current injected into it is denoted by $i_{\mathrm{g}}$. Inverters are connected to the distribution network at buses 1 and 2 , and for simplicity of exposition, we assume they have identical filter parameters and controller gains. Numerical values of the network impedances and inverter (filter and controller) parameters are listed in Table 1. Furthermore, we suppose that inverters are rated for 


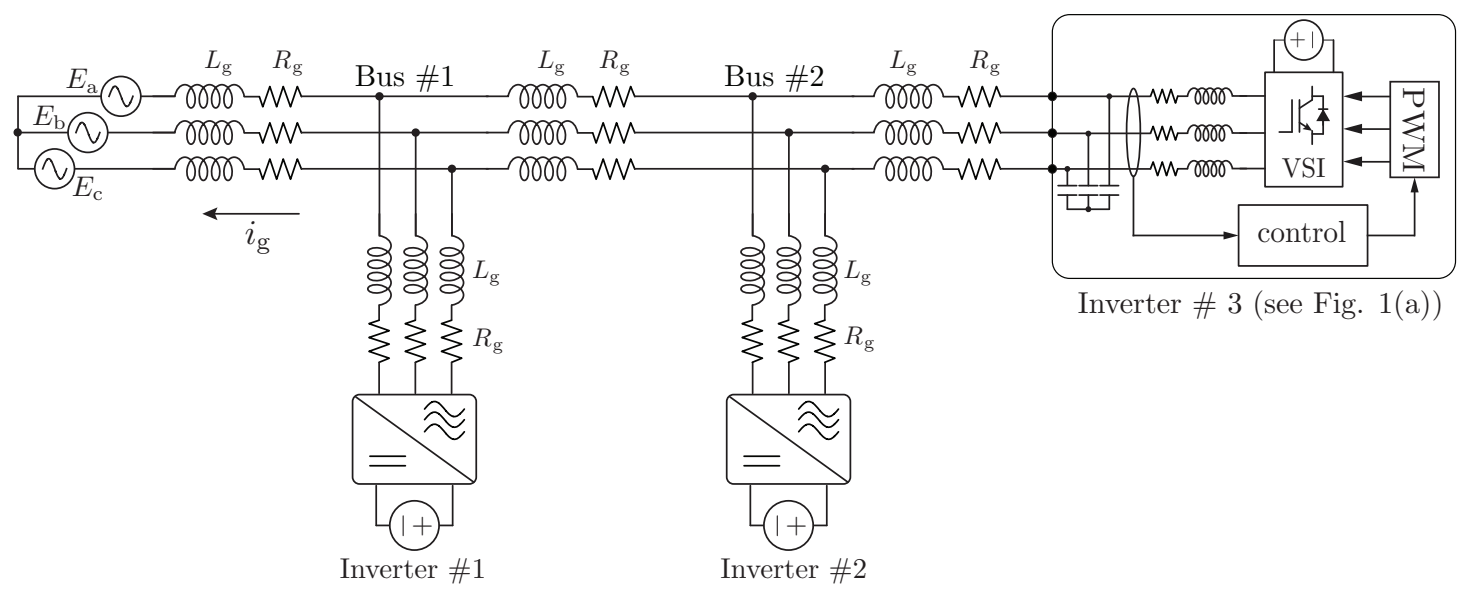

(a) switched model

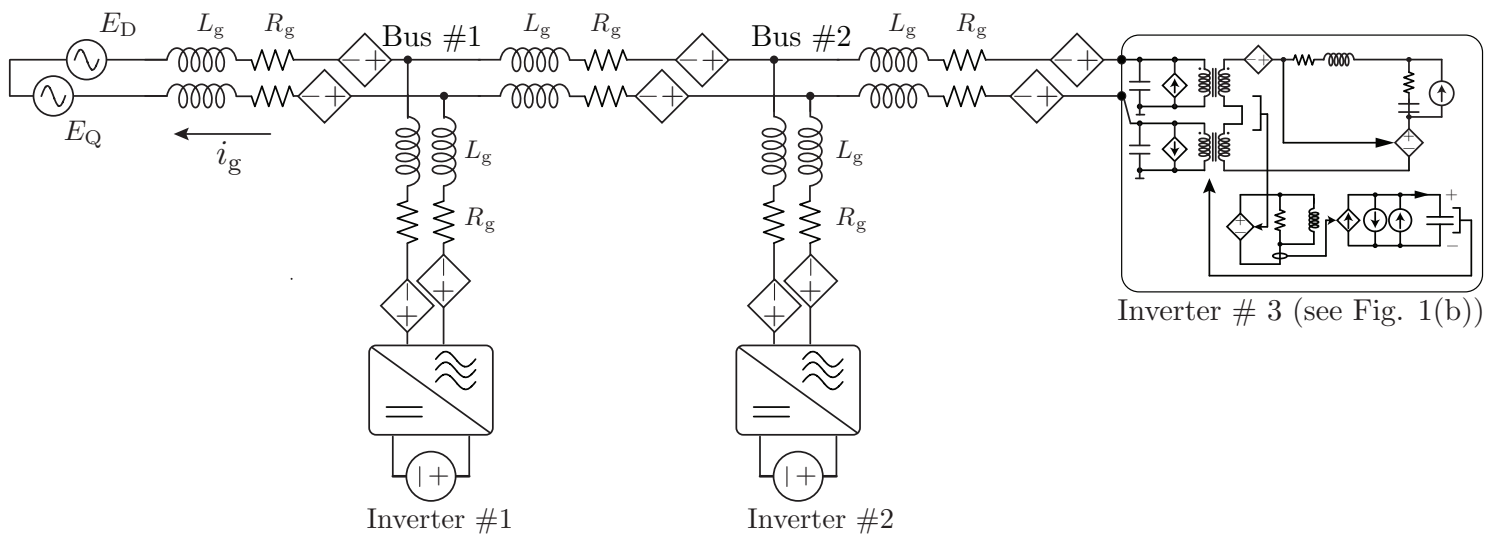

(b) averaged model

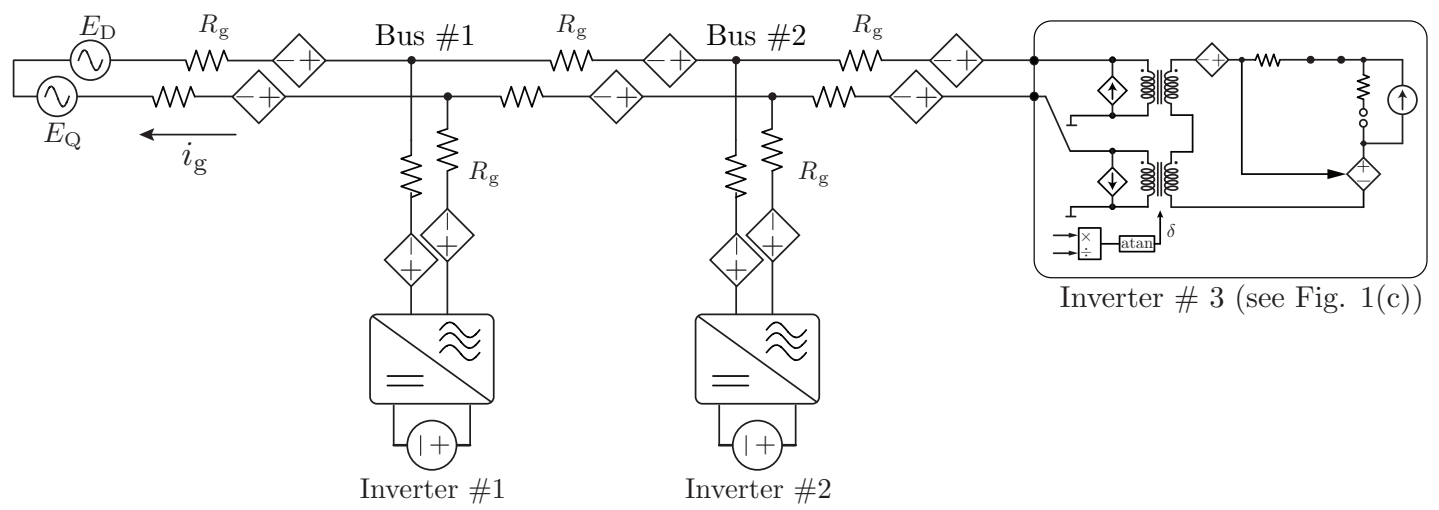

(c) steady-state model

Figure 3: Distribution network composed of three inverters connected to an infinite bus utilized for the simulation studies. (a) Detailed high-fidelity switching model where all signals are represented as sinusoids. The model for each inverter (inset sketches some features for one of the three) follows from Fig. 1(a). There is a clear distinction between physical-layer and control-layer dynamics. (b) Averaged model invokes the equivalent-circuit representation for inverter physical- and control-layer dynamics shown in Fig. 1(b). (c) Steady-state model returns system states as phasors valid in sinusoidal steady state and is built with the equivalent-circuit model shown in Fig. 1(c).

$1000[\mathrm{VA}]$ each, and the nominal RMS line-line voltage for the network is $208[\mathrm{~V}]$. Figure 3(a) aligns with prototypical representations where the power network, control loops, and switched power stage are shown as 
distinct subsystems.

The corresponding representations when inverters and their control loops are modeled as circuit equivalents are illustrated in Figs. 3(b)-(c). Figure 3(b) illustrates the averaged dynamical circuit equivalent for the inverter-based system while Fig. 3(c) corresponds to its steady-state circuit equivalent. Signals from the dynamical model in Fig. 3(b) capture dynamics at time scales where the switching behavior of inverters are disregarded. In contrast, the solution of the steady-state circuit in Fig. 3(c) returns phasors that correspond to steady-state operation. (In essence, this is the power-flow solution for the network.) In each case, network-related signals are represented in the global DQ reference frame and the ideal transformers translate signals between the global and local inverter-level dq reference frames. There is a dependent voltage source in each of the $R L$ branches in the network to capture the cross-coupling terms in the current dynamics. (We refrain from labelling these explicitly to contain notation.) Note that inductors pertaining to the network impedances are short circuited to capture steady-state operation in Fig. 3(c).

\subsection{Simulation Results}

In the simulation, the current references for the inverters are nudged with step changes as follows: (i) a step change in the current reference for inverter \#1 occurs at $0.05 \mathrm{~s}: i_{\mathrm{d}}^{\star}=5 \mathrm{~A}$ for $0 \leq t<0.05 \mathrm{~s}$ and $15 \mathrm{~A}$ for $0.05 \leq t \leq 0.20 \mathrm{~s}$; (ii) inverter \#2 experiences a current reference step change at $0.10 \mathrm{~s}: i_{\mathrm{d}}^{\star}=0 \mathrm{~A}$ for $0 \leq t<0.10 \mathrm{~s}$ and $10 \mathrm{~A}$ for $0.10 \leq t \leq 0.20 \mathrm{~s}$; (iii) a reference change occurs at inverter $\# 3$ at $0.15 \mathrm{~s}$, $i_{\mathrm{d}}^{\star}=15 \mathrm{~A}$ for $0 \leq t<0.15 \mathrm{~s}$ and $5 \mathrm{~A}$ for $0.15 \leq t \leq$

TABLE 1

Simulation Parameters for Case Studies.

\begin{tabular}{lllcl}
\hline \hline Symbol & & Description & Value & Units \\
\cline { 1 - 4 } \cline { 3 - 5 }$\omega_{\text {nom }}$ & & Nominal frequency & $2 \pi 60$ & $\mathrm{rad} / \mathrm{s}$ \\
\hline$L_{\mathrm{f}}$ & Filter inductance & 1.5 & $\mathrm{mH}$ \\
\hline$R_{\mathrm{f}}$ & Filter resistance & 0.5 & $\Omega$ \\
\hline$C_{\mathrm{f}}$ & Filter capacitance & 10 & $\mu \mathrm{F}$ \\
\hline$L_{\mathrm{g}}$ & Line inductance & 0.1 & $\mathrm{mH}$ \\
\hline$R_{\mathrm{g}}$ & Line resistance & 0.1 & $\Omega$ \\
\hline$k_{\mathrm{CC}}^{\mathrm{p}}$ & Proportional gain of CC & 2.83 & $\Omega$ \\
\hline$k_{\mathrm{CC}}^{\mathrm{i}}$ & Integral gain of CC & 942 & $\mathrm{~F}^{-1}$ \\
\hline$k_{\mathrm{PLL}}^{\mathrm{p}}$ & Proportional gain of PLL & 5 & $\Omega^{-1}$ \\
\hline$k_{\mathrm{PLL}}^{\mathrm{i}}$ & Integral gain of PLL & 10 & $\mathrm{H}^{-1}$ \\
\hline \hline
\end{tabular}

$0.20 \mathrm{~s}$. The q-component current reference, $i_{\mathrm{q}}^{\star}$, for all three inverters are held fixed at $0 \mathrm{~A}$.

Figure 4 compares the currents injected to the infinite bus for all three models. For the model which includes switched dynamics, the phase-a current is plotted in the figure. For the circuit-equivalent models (averaged and steady-state), we show the amplitude of the current. As we can see in the figure, the averaged model captures the amplitude of the switched model accurately, while the amplitude in the steady-state model is changed to the steady-state value of the switched model at every step change. Note that the averaged model can reproduce the sinusoidal trajectories with an appropriate inverse abc-to-dq transformation. In other words, the averaged model can reproduce all dynamics of the switched model with the exception of switching ripple.

Figures 5 and 6 show the voltage amplitudes and phases for bus $\# 1$ and bus $\# 2$, respectively. We observe that the voltage amplitudes and phases for the three model types are in close agreement and differences clearly reflect the differing fidelity of each model flavor. Note that the solution corresponding to the steady-state circuit would be conventionally obtained by solving a power-flow problem and it is only valid in sinusoidal steady state. Next, Fig. 7 compares the phase angle difference, $\delta$, between the global DQ frame and the local frame for inverter \#1. Furthermore, Fig. 8 shows the output current from inverter \#1 for each model type. Evidently, we see that the steady-state circuit can provide the steady-state solution with time series input data (i.e., current references in our case study).

To provide a comprehensive view on accuracy, we plot the error in the averaged simulation and steady-state simulation (with respect to suitable baselines) for all voltage magnitudes and phase angles in the network in Figs. 9 and 10. The maximum error in voltages across the simulation horizon and all buses is $2 \mathrm{~V}$

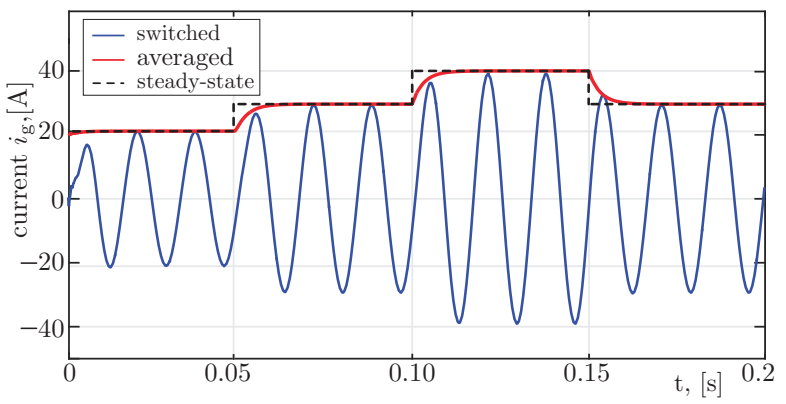

Figure 4: Current injected into the infinite bus. The result for the switched model shows the phase-a current, while the results for the averaged and steady-state circuit models show the current amplitude. 

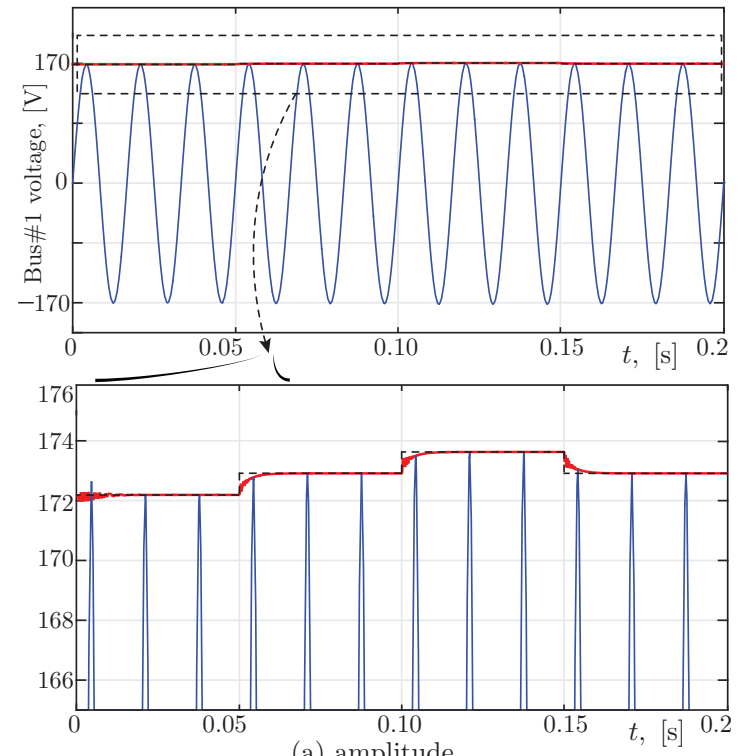

(a) amplitude

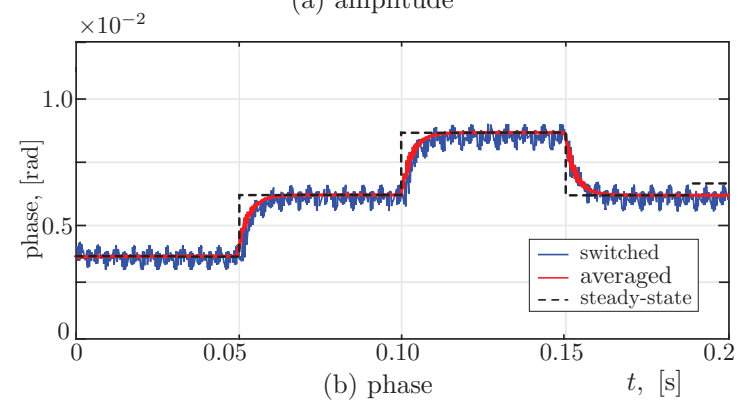

Figure 5: Voltage at Bus \#1: (a) amplitude and inset showing details, (b) phase.

(120 Vrms nominal voltage), and the maximum error in phase angles across the simulation horizon and all buses is $0.5 \times 10^{-3}$ rad. The computation times for the $0.2 \mathrm{~s}$ simulation horizon for the switched, averaged, and steady-state models are $22.49 \mathrm{~s}, 0.25 \mathrm{~s}$, and $0.15 \mathrm{~s}$, respectively. As expected, the averaged and steady-state models can be simulated much faster while retaining most essential dynamical attributes pertinent to synchronous operation. Furthermore, this also suggests that the steady-state equivalent circuit model can be utilized to rapidly initialize detailed switch-level simulation models if necessary.

\section{Conclusions and Future Work}

In this paper, we demonstrated how systems of interconnected current-controlled inverters can be modeled as a unified equivalent-circuit network. To establish a system-wide circuit equivalent network, we translated all relevant physics and control loops as circuits. Ultimately, two circuit-based models were
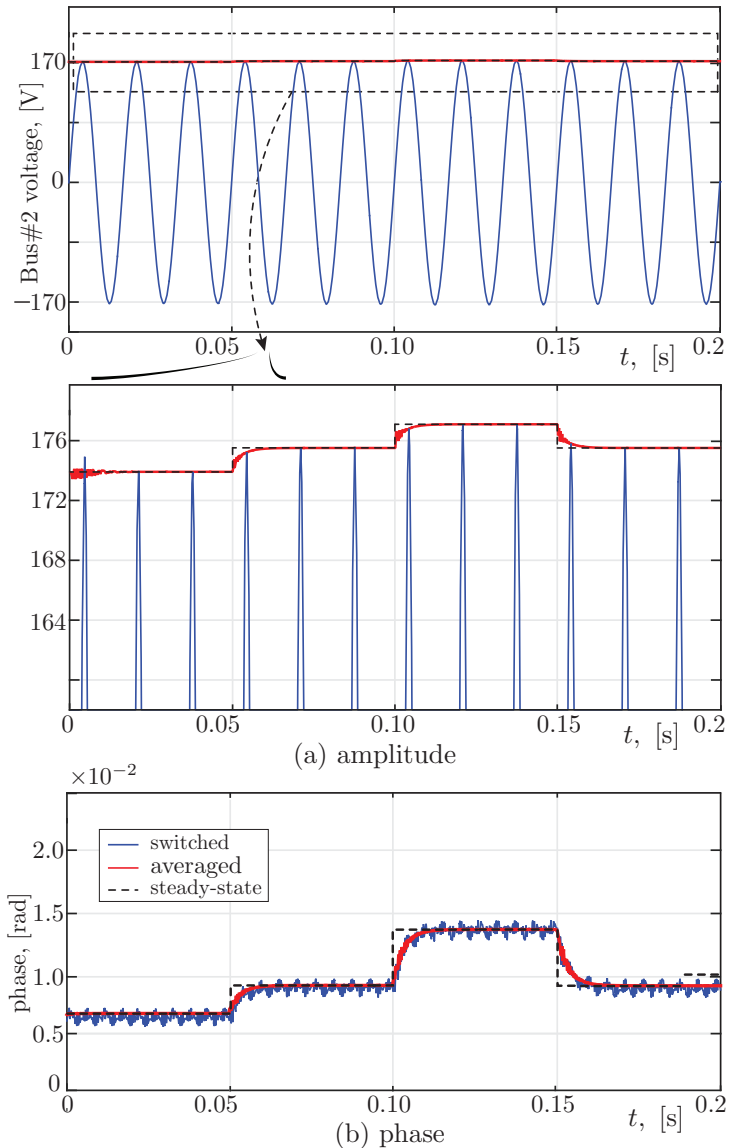

Figure 6: Voltage at Bus \#2: (a) amplitude and inset showing details, (b) phase.

put forth for computing the system equilibrium and analyzing nonlinear time-domain dynamics. Future work involves scaling the approach to larger and possibly unbalanced networks with more detailed and involved models for inverters that have different control types (grid-following and grid-forming). We believe

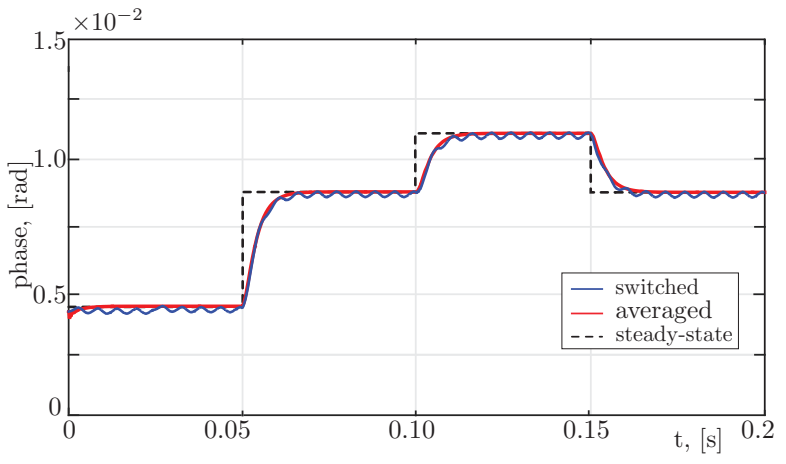

Figure 7: Dynamics of phase angle $\delta$ for inverter \#1. 


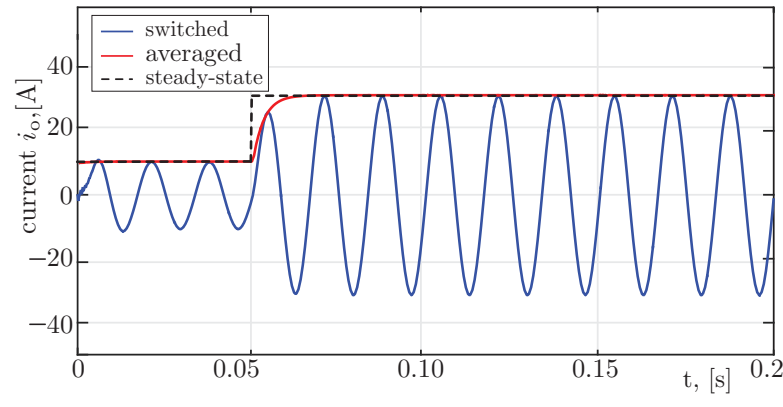

Figure 8: Output current for inverter \#1, $i_{\mathrm{o}}$.

similar models for other energy-conversion interfaces such as synchronous generators, induction machines, and energy storage devices can also be incorporated alongside inverters in a unified setting.

\section{References}

[1] M. Prodanović and T. C. Green, "Control and filter design of three-phase inverters for high power quality grid connection," IEEE Transactions on Power Electronics, vol. 18, pp. 373-380, Jan. 2003.

[2] N. Pogaku, M. Prodanović, and T. C. Green, "Modeling, analysis and testing of autonomous operation of an inverter-based microgrid," IEEE Transactions on Power Electronics, vol. 22, pp. 613-625, Mar. 2007.

[3] V. Purba, S. V. Dhople, S. Jafarpour, F. Bullo, and B. B. Johnson, "Reduced-order structure-preserving model for parallel-connected three-phase grid-tied inverters," in IEEE 18th Workshop on Control and Modeling for Power Electronics (COMPEL), pp. 1-7, Jul. 2017.

[4] D. C. Meeker, E. H. Maslen, and M. D. Noh, "An augmented circuit model for magnetic bearings including eddy currents, fringing, and leakage," IEEE Transactions on Magnetics, vol. 32, pp. 3219-3227, Jul. 1996.

[5] M. Cheng, K. T. Chau, and C. C. Chan, "Static characteristics of a new doubly salient permanent magnet motor," IEEE Transactions on Energy Conversion, vol. 16, pp. 20-25, Mar. 2001.

[6] Z. Q. Zhu, Y. Pang, D. Howe, S. Iwasaki, R. Deodhar, and A. Pride, "Analysis of electromagnetic performance of flux-switching permanent-magnet machines by nonlinear adaptive lumped parameter magnetic circuit model," IEEE Transactions on Magnetics, vol. 41, pp. 4277-4287, Nov. 2005.

[7] G. R. Slemon, "An equivalent circuit approach to analysis of synchronous machines with saliency and saturation," IEEE Transactions on Energy Conversion, vol. 5, pp. 538-545, Sep. 1990.

[8] J. D. Law, T. J. Busch, and T. A. Lipo, "Magnetic circuit modelling of the field regulated reluctance machine. Part I: model development," IEEE Transactions on Energy Conversion, vol. 11, pp. 49-55, Mar. 1996.

[9] L. Wang, J. Jatskevich, and H. W. Dommel, "Re-examination of synchronous machine modeling techniques for electromagnetic transient simulations," IEEE Transactions on Power Systems, vol. 22, pp. 1221-1230, Aug. 2007.
[10] L. Wang, J. Jatskevich, and S. D. Pekarek, "Modeling of induction machines using a voltage-behind-reactance formulation," IEEE Transactions on Energy Conversion, vol. 23, pp. 382-392, Jun. 2008.

[11] R. Middlebrook, "Modeling current-programmed buck and boost regulators," IEEE Transactions on Power Electronics, vol. 4, pp. 36-52, Jan. 1989.

[12] M. K. Kazimierczuk and A. J. Edstrom, "Open-loop peak voltage feedforward control of PWM buck converter," IEEE Transactions on Circuits and Systems I: Fundamental Theory and Applications, vol. 47, pp. 740-746, May 2000.

[13] B. Bryant and M. K. Kazimierczuk, "Open-loop power-stage transfer functions relevant to current-mode control of boost PWM converter operating in CCM," IEEE Transactions on Circuits and Systems I: Regular Papers, vol. 52, pp. 2158-2164, Oct. 2005.

[14] J. Sun, "Impedance-based stability criterion for grid-connected inverters," IEEE Transactions on Power Electronics, vol. 26, pp. 3075-3078, Nov. 2011.

[15] L. Harnefors, M. Bongiorno, and S. Lundberg, "Input-admittance calculation and shaping for controlled voltage-source converters," IEEE Transactions on Industrial Electronics, vol. 54, pp. 3323-3334, Dec. 2007.

[16] J. Sun, "Small-signal methods for AC distributed power systems - A review," IEEE Transactions on Power Electronics, vol. 24, pp. 2545-2554, Nov. 2009.

[17] J. He and Y. W. Li, "Generalized closed-loop control schemes with embedded virtual impedances for voltage source converters with LC or LCL filters," IEEE Transactions on Power Electronics, vol. 27, pp. 1850-1861, Apr. 2012.

[18] X. Wang, L. Harnefors, and F. Blaabjerg, "Unified impedance model of grid-connected voltage-source converters," IEEE Transactions on Power Electronics, vol. 33, pp. 1775-1787, Feb. 2018.

[19] L. A. B. Tôrres, J. P. Hespanha, and J. Moehlis, "Power supplies dynamical synchronization without communication," in 2012 IEEE Power and Energy Society General Meeting, Jul. 2012.

[20] B. Johnson, S. V. Dhople, A. O. Hamadeh, and P. T. Krein, "Synchronization of Nonlinear Oscillators in an LTI Electrical Power Network," IEEE Transactions on Circuits and Systems I: Regular Papers, vol. 61, pp. 834-844, Mar. 2014.

[21] B. Johnson, M. Sinha, N. Ainsworth, F. Dörfler, and S. Dhople, "Synthesizing Virtual Oscillators to Control Islanded Inverters," IEEE Transactions on Power Electronics, vol. 31, pp. 6002-6015, Aug. 2016.

[22] M. Lu, S. Dutta, V. Purba, S. Dhople, and B. Johnson, "A grid-compatible virtual oscillator controller: Analysis and design," in 2019 IEEE Energy Conversion Congress and Exposition (ECCE), pp. 2643-2649, Sep. 2019.

[23] M. Colombino, D. Groß, J. Brouillon, and F. Dörfler, "Global phase and magnitude synchronization of coupled oscillators with application to the control of grid-forming power inverters," IEEE Transactions on Automatic Control, vol. 64, no. 11, pp. 4496-4511, 2019.

[24] Z. Shi, J. Li, H. I. Nurdin, and J. E. Fletcher, "Comparison of virtual oscillator and droop controlled islanded three-phase microgrids," IEEE Transactions on Energy Conversion, vol. 34, pp. 1769-1780, Dec. 2019. 


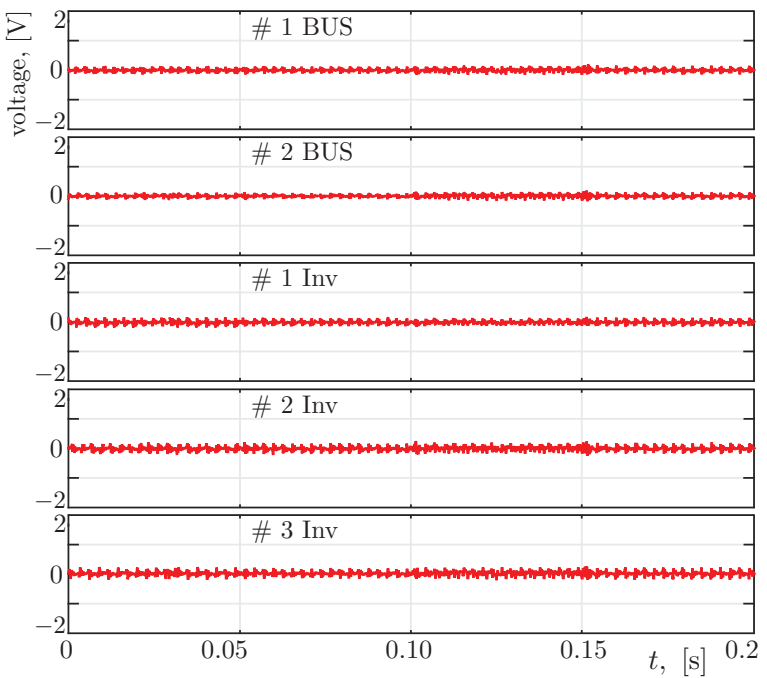

(a) errors, switched model - average model

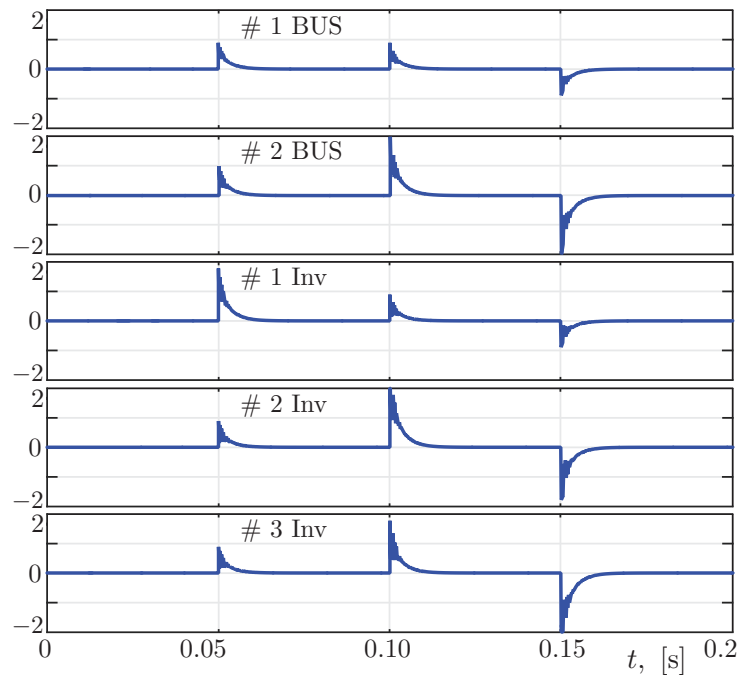

(b) errors, steady-state model - average model

Figure 9: Error in voltage magnitudes.

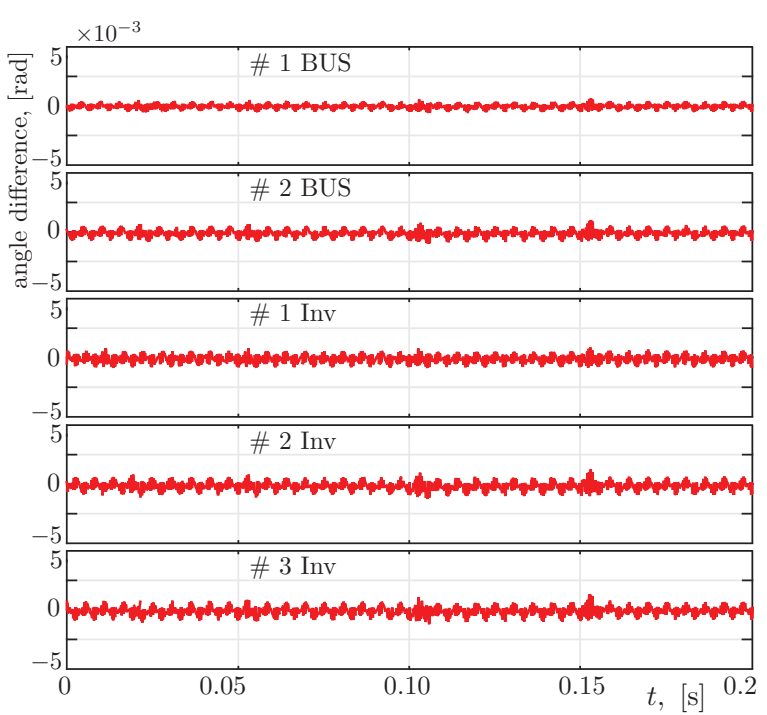

(a) errors, switched model - average model

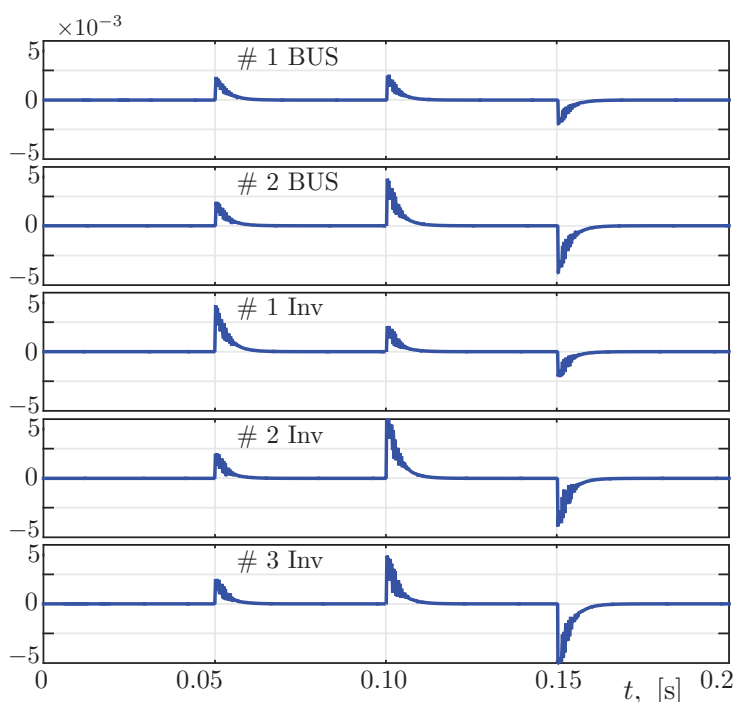

(b) errors, steady-state model - average model

Figure 10: Error in phase angles.

[25] R. Mallik, B. Majmunović, S. Mukherjee, S. Dutta, G. Seo, D. Maksimović, and B. Johnson, "Equivalent circuit models of voltage-controlled dual active bridge converters," in IEEE 20th Workshop on Control and Modeling for Power Electronics (COMPEL), pp. 1-4, 2019.

[26] B. Johnson, M. Lu, V. Purba, and S. Dhople, "Circuit-equivalent models for current-controlled inverters," in IEEE 20th Workshop on Control and Modeling for Power Electronics (COMPEL), pp. 1-5, 2019.

[27] A. Yazdani and R. Iravani, Voltage-Sourced Converters in Power Systems. Hoboken, NJ: John Wiley \& Sons, Inc., 2010.

[28] E. Twining and D. G. Holmes, "Grid current regulation of a three-phase voltage source inverter with an LCL input filter," IEEE Transactions on Power Electronics, vol. 18, pp. 888-895, May 2003.

[29] C. A. Plet, M. Graovac, T. C. Green, and R. Iravani, "Fault response of grid-connected inverter dominated networks," in IEEE PES General Meeting, pp. 1-8, Jul. 2010.

[30] N. Bottrell, M. Prodanović, and T. C. Green, "Analysed small signal state-space model of an active rectifier," in 45th International Universities Power Engineering Conference (UPEC), pp. 1-6, 2010. 